\title{
A diabetic gentleman who strimmed his way to DKA
}

\author{
KRISTINA MK LEE, KERRI BAKER
}

\section{Introduction}

A healthy, active 75 year-old man, diagnosed with diabetes nearly 37 years earlier, presented with his first episode of DKA. He lived alone and for the previous two days, due to non-specific weakness and pain - particularly in the hip region - had been confined to bed and unable to access food, fluid or insulin. A detailed history and careful scrutiny of investigational images permitted diagnosis of acetabular fractures.

\section{Case Report}

A 75 year-old gentleman with normally well controlled insulin dependent diabetes presented to Accident and Emergency department with vomiting, weakness and two episodes of collapse. He otherwise felt well, although looked clinically dehydrated. His random blood glucose of $38 \mathrm{mmol} / \mathrm{l},++++$ urinary ketones and a venous blood gas pH of 7.27, confirmed the diagnosis of DKA and he was subsequently managed according to the local DKA protocol.

This was the patient's first presentation with DKA, having been diagnosed with diabetes at the age of 38. He had been seen in the diabetes clinic two months earlier and had been described to have stable diabetes. The patient had no significant past medical history other than hypertension.

On further questioning to elucidate the cause of this episode, the patient revealed that a week prior to presentation he had carried out some heavy gardening, strimming over six acres of land. This, along with a further two days of long distance walking at the Edinburgh Jazz Festival, had resulted in non-specific weakness and widespread musculoskeletal pain, particularly around his hips. As a consequence, he had been unable to get out of bed and, as he lived alone, could not access food or drink and had not taken any insulin during the two days prior to presentation.

Due to the significant hip and lateral thigh pain, a pelvic $x$ ray was performed which demonstrated no obvious abnormalities (Figure 1). However, due to the clinical suspicion of acetabular fractures due to reduced range of motion of both

Department of Acute Medicine, Victoria Hospital, Kirkcaldy, KY2 5AH, UK

Address for correspondence: Dr Kristina Lee

Department of Acute Medicine, Victoria Hospital, Kirkcaldy, KY2 5AH, UK.

Tel: +44 (0)1592 643355

E-mail: klee@doctors.org.uk

Br J Diabetes Vasc Dis 2014;14:75-76

http://dx.doi.org/10.15277/bjdvd.2014.013

\author{
Abbreviations and acronyms \\ CT computerised tomography \\ DKA diabetic ketoacidosis
}

Figure 1. Pelvic $X$ ray demonstrating no evidence of acetabular fractures.

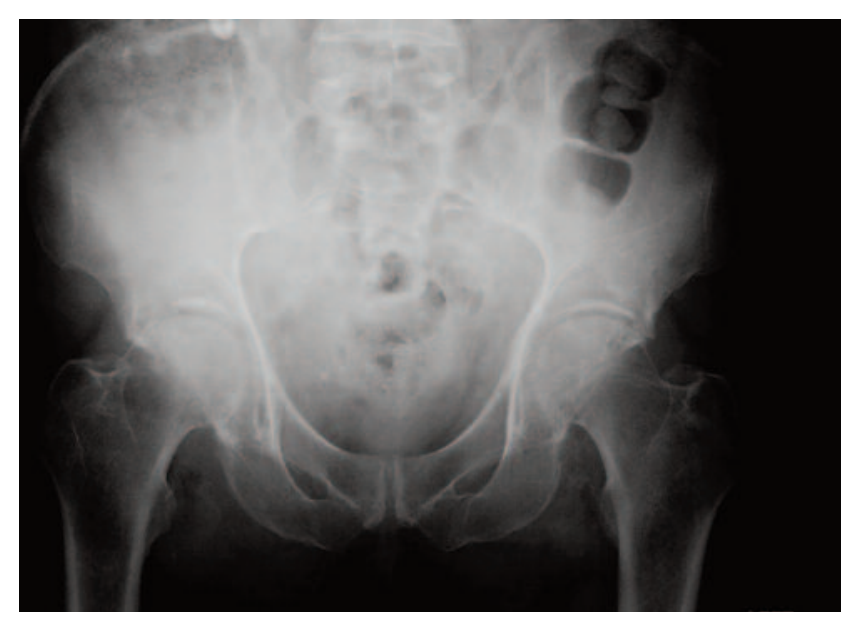

hips on clinical examination, further imaging was sought. CT pelvis revealed a left minimally displaced acetabular fracture involving both columns and ilium and a small, minimally displaced right acetabular fracture (Figure 2). Other possible differentials included myopathy and malignant causes of fracture but were excluded by normal creatine kinase, lactate and calcium levels.

The final diagnosis was documented as DKA due to reduced insulin intake secondary to spontaneous bilateral acetabular fractures during heaving garden strimming. The fractures are currently being managed conservatively with analgesia and ongoing physiotherapy and rehabilitation. A complete recovery is expected and he has since returned to his pre-admission insulin regimen.

\section{Discussion}

DKA is a life threatening condition and early recognition is essential in order for prompt life-saving therapy to be initiated. Correctly identifying and treating precipitating causes of DKA is imperative to help ensure the most appropriate management for the patient. It also provides an opportunity to educate the patient in recognising and preventing future episodes, for example 
Figure 2. CT scan of pelvis demonstrating left minimally displaced acetabular fracture involving both columns and ilium and a small, minimally displaced right acetabular fracture. Green lines to aid orientation of the various views. a) horizontal plane b) coronal plane c) sagittal plane

a

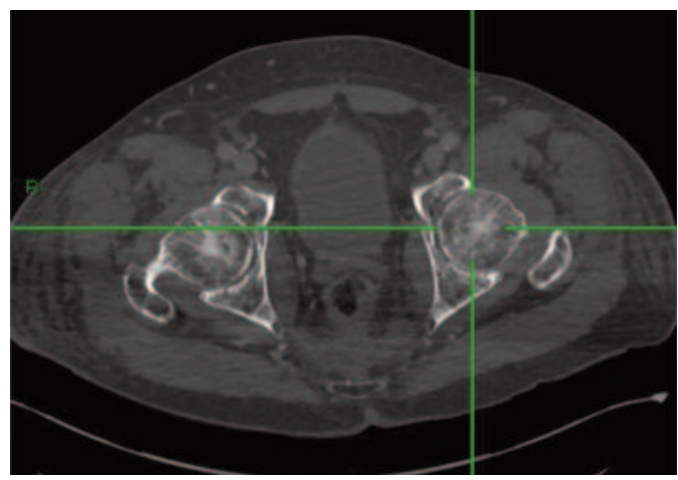

b

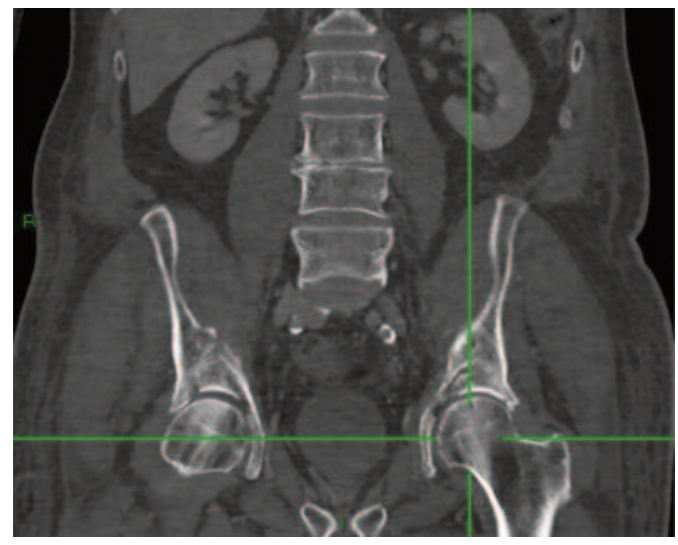

C

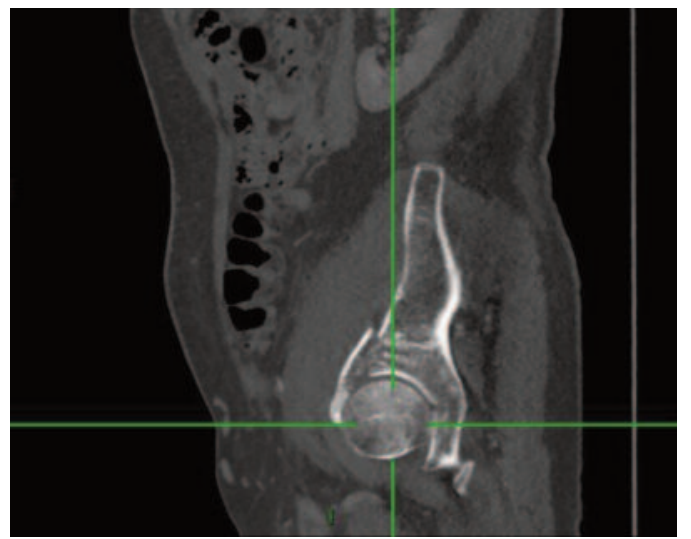

by reiterating 'sick day 'rules. ${ }^{1}$ For many, infection is the most common precipitating factor, but other causes include other intercurrent illness, psychological stress and omission of insulin. In this case, we present an unusual cause for this due to spontaneous bilateral acetabular fractures. ${ }^{2}$

Acetabular stress fractures are rare and, in the literature, appear to occur in populations who experience repeated high impact such as military or endurance athletes, particularly in

\section{Key messages}

- Identifying and treating precipitating causes of DKA is an essential part of the management.

- Ensuring access to insulin is important, particular in patients who live alone or may be prone to injury or illness which may render them unable to access insulin or monitor blood sugar levels.

- In cases where there is a high clinical suspicion of acetabular fracture, further imaging should be sought as they can be commonly missed on plain radiographs.

those who concomitantly have with low bone mineral density. ${ }^{3}$ Acetabular stress fractures, particularly bilaterally, are almost unheard of in our patient's demographic, particularly as there was no history of previous hip surgery or falls. ${ }^{4}$ Although initial imaging suggested no fractures, acetabular fractures are commonly missed on x-ray and therefore warrant further imaging. ${ }^{5}$

To our knowledge, this is the first case of bilateral acetabular fractures secondary to heavy strimming, and this case presents a rare and unusual cause for DKA. Although it is highly unlikely that our patient will be incapacitated by acetabular fractures again, it highlights the importance of ensuring access to insulin, particularly in those who live alone or those who may be more prone to injury or illness which may render the patient unable to access their insulin or monitor their blood sugar levels. By correctly identifying precipitating causes we can ensure appropriate management for the underlying problem and enable appropriate changes to prevent future episodes.

\section{Conflict of interest None \\ Funding sources None}

Acknowledgements The authors would like to thank Dr Thomas Hartley, consultant radiologist at the Victoria Hospital, Kirkcaldy, for his technical assistance with the radiological images.

\section{References}

1. Savage MW, Dhatariya KK, Rayman G et al. Joint British Diabetes Societies guideline for the management of diabetic ketoacidosis. Diabet Med 2011;28:508-15. http://dx.doi.org/10.1111/j.1464-5491.2011.03246.x

2. Kitabchi $A E$, Umpierrex $G E$, Murphy $M B$ et al. Hyperglycemic crises in diabetes. Diabetes Care 2004:27(suppl 1):s94-s102.

http://dx.doi.org/10.2337/diacare.27.2007.594

3. Williams TR, Puckett ML, Denison $G$ et al. Acetabular stress fractures in military endurance athletes and recruits: incidence and MRI and scintigraphic findings. Skeletal Radiol 2002;31:277-81. http://dx.doi.org/10.1007/s00256-002-0485-0

4. Tornkvist $\mathrm{H}$ and Schatzker J. Acetabular fractures in the elderly: an easily missed diagnosis. J Orthop Trauma 1993;7:233-5. http://dx.doi.org/10.1097/00005131-199306000-00006

5. Senohradski L, Karovic B and Miric D. [Computer tomography in the diagnosis and therapy of acetabular fractures]. Srp Arh Celok Lek 2001; 129:194-8. 